\title{
Intravascular lithotripsy of an underexpanded stent following unsuccessful rotational atherectomy in a patient with severely calcified coronary artery
}

Wojciech Faron $^{1 *}$, Jarosław Hiczkiewicz ${ }^{1,2 *}$, Jan Budzianowski ${ }^{1,2}$, Maciej Lesiak ${ }^{3}$

${ }^{1}$ Department of Cardiology, Nowa Sol Hospital, Nowa Sol, Poland

${ }^{2}$ Collegium Medicum, University of Zielona Gora, Zielona Gora, Poland

${ }^{3} 1^{\text {st }}$ Department of Cardiology, Poznan University of Medical Sciences, Poznan, Poland

A 66-year-old man with hypertension was admitted to hospital with non-ST-segment elevation myocardial infarction. Emergency coronary angiography demonstrated critical stenosis in the medial and distal segment of the right coronary artery (RCA) (Fig. 1A). During the same procedure rotational atherectomy was unsuccessfully attempted, because the RotaWire guide wire (Boston Scientific, Marlborough, USA) did not reach the distal part of the RCA. One month later another RCA rotational atherectomy was attempted using the RotaLink System (Boston Scientific, Marlborough, USA). Rotablation with 1.5 burr was performed followed by predilatation with two noncompliant (NC) balloons $(2.5 \times 20 \mathrm{~mm})$ and $(3.0 \times 20 \mathrm{~mm})$ (Fig. 1B). Afterwards, two drug-eluting stents (Onyx, USA) $(3.0 \times 30 \mathrm{~mm})$ distally and $(3.5 \times 38 \mathrm{~mm})$ proximally were implanted. Despite postdilatation with $\mathrm{NC}$ balloons $(3.5 \times 12 \mathrm{~mm}, 14 \mathrm{~atm}$ and $4.0 \times$ $\times 12 \mathrm{~mm}, 24 \mathrm{~atm})$ it was not possible to expand the proximal stent optimally (Fig. 1C).
In the next step, we decided to attempt adjunctive intravascular lithotripsy for stent optimalization in the proximal RCA. Shockwave balloon was delivered in the underexpanded stent and 8 rounds of 10 pulses were applied (with balloon inflation at 2-4-6 atm) (Fig. 1D). Postdilatation with a NC high-pressure balloon $4.0 \times 12 \mathrm{~mm}$ (infl. 18-20 atm) was used to optimize the final result (Fig. 1E). The angiography confirmed significant expansion of the implanted stent with residual $20 \%$ diameter stenosis (Fig. 1F).

Rotational atherectomy was performed to reduce the volume of calcium in the vessel and to prepare it for the stent implantation. However, massive calcification of the RCA was a major obstacle for the optimal stent expansion despite NC balloons for the postdilatation applied. The intravascular lithotripsy procedure is an alternative for the management of stent underexpansion due to calcific coronary artery disease.

Conflict of interest: None declared

Address for correspondence: Jan Budzianowski, MD, PhD, Collegium Medicum, University of Zielona Gora, ul. Zyty 28, 65-046 Zielona Góra, Poland, tel: +48 683882 103, e-mail: jbudzianowski@uz.zgora.pl

Received: 21.02.2021 Accepted: 5.05.2021

*Equally contributed

This article is available in open access under Creative Common Attribution-Non-Commercial-No Derivatives 4.0 International (CC BY-NC-ND 4.0) license, allowing to download articles and share them with others as long as they credit the authors and the publisher, but without permission to change them in any way or use them commercially. 


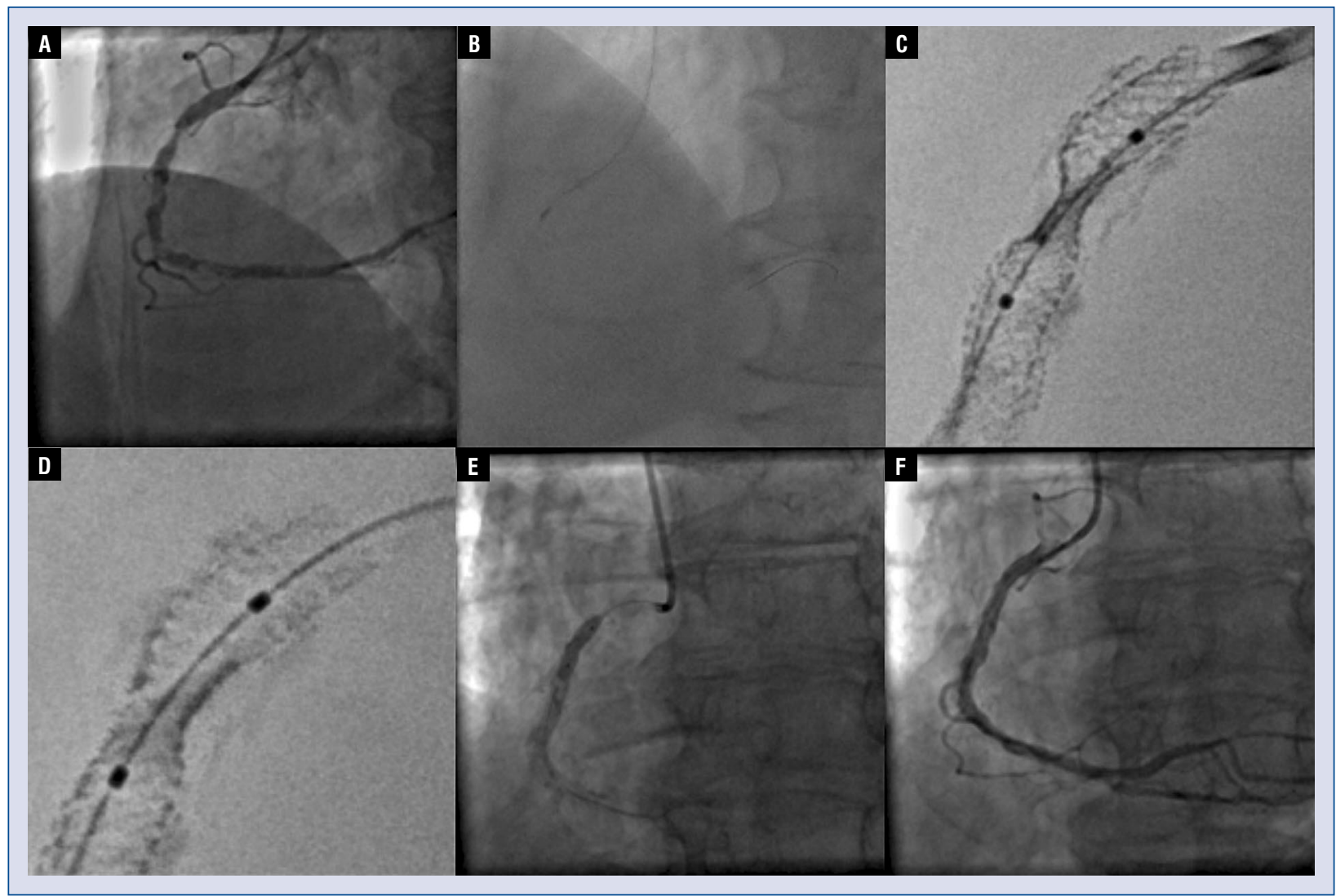

Figure 1. A. Severely calcified artery disease at the medial and distal part of the right coronary artery (RCA); B. Rotational atherectomy with 1.5 burr; C. Underexpanded proximal stent in the RCA - CLEAR stent view; D. Angiography after intravascular lithotripsy on CLEAR stent visualization; E. Postdilatation with 4.0 noncompliant high-pressure balloon; F. Final angiographic result. 